\title{
iaBastos: An Intelligent Marketplace for Agricultural Products
}

\author{
Gonzalo A. Aranda-Corral ${ }^{1}$, Joaquín Borrego Díaz², and \\ David Solís Martín ${ }^{2}$ \\ 1 Depto. de Tecnologías de la Información, Universidad de Huelva, \\ Crta. Palos de La Frontera s/n, 21819 Palos de La Frontera, Spain \\ garanda@us.es \\ 2 Depto. de Ciencias de la Computación e Inteligencia Artificial, \\ Universidad de Sevilla, Avda. Reina Mercedes s/n, 41012 Sevilla, Spain
}

\begin{abstract}
In this paper we present a multi-agent platform designed to support p2p auctions on agricultural products. Experiments show that it could be feasible to integrate a platform, which is de-centralized by nature, into the current socioeconomic environment. The reason is that this market is managed by hard-centralized ecosystem, and experiments with our system show a significant reduction in marketing margins for the benefit of consumers and producers.
\end{abstract}

\section{Introduction and Motivation}

In Spain, the existence of some issues in the relation between producers and intermediaries in agricultural markets is well known. One can find a large number of news that reveal the significant price increase that takes place since the product leaves the production zone until it arrives to final consumers. This increase not only affects to final consumers, but also producers are warning about the low income that they get, which even could not cover production costs.

This rigid business ecosystem contrasts with the astonishing number of transactions through $\mathrm{P} 2 \mathrm{P}$ auctions, applied to very different goods and services by means of Internet. For example, in 2001, eBay had 42 million users and it auctioned 1 million items per day. Internet auctions are available to anyone with a computer or mobile and an internet connection [2].

In this paper, we propose a Multiagent System (MAS), called iaBastos, to provide an infrastructure to support auctions between agricultural producers and final consumers (or retailers). More specifically, in this work we study if it is affordable to decentralize hard-centralized markets.

Within the scope of similar markets, the use of auctions in fish markets is well known. For example MASFIT [1] is a system where software agents live together with human agents in the auction process of fish. In the scope of agri-cultural products, it is usual to find only sites like http://www.arcocoag.org or http:// www.naranjasyfrutas.com, where users can search producers for a specific product, which aims to bring that two profiles closer. 



Fig. 1. left: MAS architecture, right: MAS ecosystem associated to iaBasto

\section{2 iaBastos' Architecture}

The iaBastos platform provides support to a system more complex than a simple contact directory between producers and consumers; it is a complete and autonomous auction system. Unlike MASFIT system, in this platform, there is not any integration between human and software agents within the auction process, in which only take part software agents (see Fig. 1).

The architecture has five main modules (see Fig. 1 left):

- Database: Django's ORM allows to abstract the database management.

- Business module: Implements business logic (user management, authoring and authentication) and interacts with the Database module.

- REST web services: allow the MAS to interact with the business module.

- MAS: Developed using JADE platform [5] (http://www.jade.tilab.com) and FIPA English Auction Interaction Protocol Specification (http://www. fipa.org/specs/fipa00031/). It performs the autonomous auction process.

- Web and Android app: provides an user interface where auction rules can be managed, and achieved contracts can be looked up. The android app also provides instantaneous notifications and warnings on smartphones and tablets.

Another key difference with respect to MASFIT -and other auction systemsis that in our case we have three target profiles, buyers, sellers and carriers (those providing logistics). To solve this issue we have to split the auction process in two semi-independent auctions as it is shown in Fig. 1 (right). When the auction system runs, it takes all rules, defined by different profiles, into the system. Randomly, it takes the first farmer rule and starts an auction to find a buyer. All buyers have to consider how much money they must save in order to find a transport. After the auction between the farmer and buyers finishes, if a deal is reached, the auction between the buyer and carriers starts. If an agreement 
is reached in both auctions, users will be notified and the system will continue with the next farmer. Otherwise, the previous deal between farmer and buyer will be dismissed. The auction system will continue working until any new deal can be found.

Therefore, we can find the following software agents:

- SynAgent: this agent is responsible for getting all available rules through the web appropriate service.

- BuyerRuleAgent: this agent represents a buyer rule and its mission is to buy a product taking into account the constraints.

- SaleRuleAgent: this agent is responsible for selling the farmer products.

- CarrierRuleAgent: this agent represents the carrier's behavior in order to get a transport contract.

- BuyerAndFarmerDeal: this agent tries to find transport after a buyer has achieved a deal with a farmer.

\section{Experimentation}

As we mentioned earlier, three profiles can be found in our system (see Figure 1). After the registration process, the user has to set the rules based on his profile. Farmers has to set products (from harvests) as well as sale rules (based on quality, minimum sale price, minimum weight for orders, etc.); buyers has to set the buyer rules (based on quality product, maximum buying price, kilogram range, ...) and carriers in the same way (maximum weight per order, maximum distance,etc.). Once the overall rule set is in the system the MAS dynamics starts.
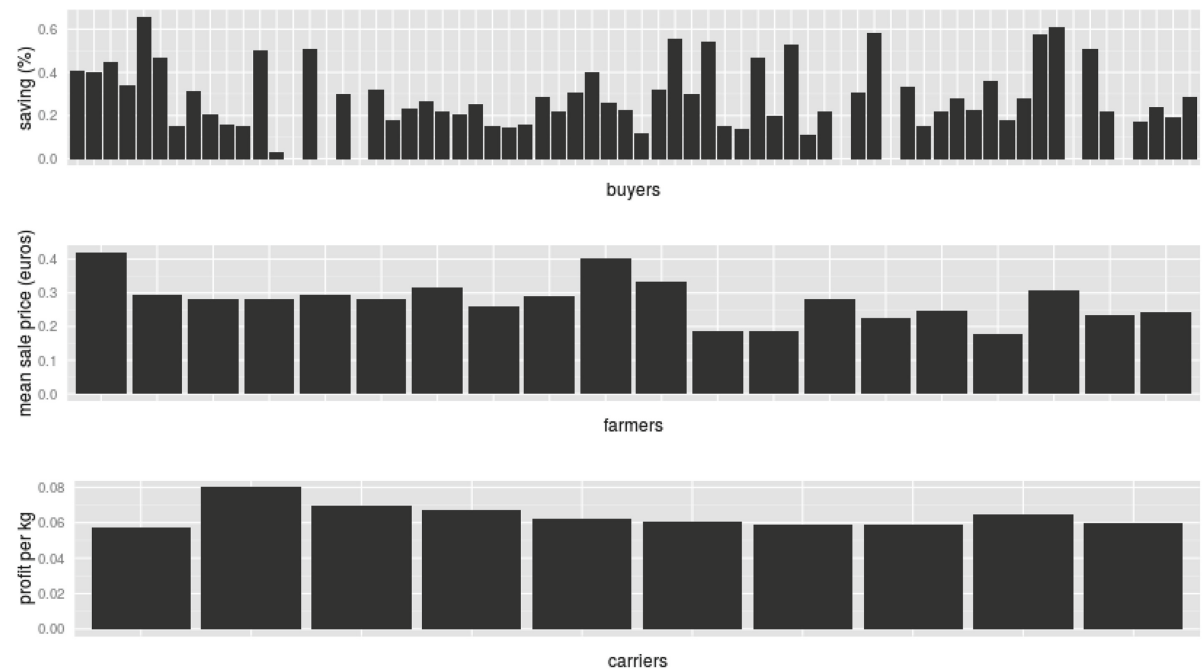

Fig. 2. up: savings obtained by each buyer, middle: mean sale price per kilogram by each farmer, down: total profit per kilogram obtained by each carrier 
For demonstration purposes, we have created an experimental set of producers, consumers (or retailers) and carriers around the city of Seville. To be as realistic as possible, such agents have been geolocated in order to compute road distances. Farmer rules (what product, what quality and the minimum sale's price) are designed, taking minimum price a higher value than the current sale prices get by producers. Further, we have been created buyer and transport rules. We have considered 68 buyer rules, 20 farmer rules and 10 carrier rules.

When the system stops, it has got 61 deals. As shown in Figure 2, most buyers have obtained very good savings with a mean of $29 \%$. Farmers are buying its products to a mean price of 0.27 cents. This price is very higher than the current mean market. Carriers offered transport to a mean of 6 cents per kilogram, with a very low standard deviation.

\section{Conclusions}

This work presents a MAS-based auction system oriented to agricultural market. The system is composed by a website, a mobile app and the multi-agent system. Actually, it is a beta version and we are studying different agent strategies to get a more advantageous system for all profiles (farmers, buyer and carriers) and adding new functionalities to the website. For example, to give carriers tools for managing his routes and deliveries.

On the initial question, whether is possible to decentralize hard-centralized markets, we think that it is truly possible, we have margin on buyer savings and on sale price. The only remaining doubt is if society is ready to take this system on.

\section{References}

1. Cuni, G., Esteva, M., Garcia, P., Puertas, E., Sierra, C., Solchaga, T.: MASFIT: Multi-Agent System for Fish Trading (2004)

2. Krishna, V.: Auction Theory. Academic Press (2009)

3. Weiss, G.: Multiagent systems. MIT Press (2013)

4. Poslad, S.: Specifying Protocols for Multi-Agent Systems Interaction (2007)

5. Bellifemine, F., Poggi, A., Rimassa, G.: JADE-A FIPA-compliant agent framework (1999) 